\title{
Comparison of the Anterior and Posterior Approaches for the Management of Spinal Cord Injury: A Systematic Review
}

\author{
I Dewa Gede Bracika Damma Prasada ${ }^{1}$, Ketut Gede Mulyadi Ridia ${ }^{2}$ \\ ${ }^{1}$ Resident of Orthopaedics and Traumatology Department, ${ }^{2}$ Consultant of Orthopaedics and Traumatology \\ Department, Faculty of Medicine Udayana University, Sanglah General Hospital
}

Corresponding Author: I Dewa Gede Bracika Damma Prasada

\begin{abstract}
Introduction: Spinal cord injury (SCI) is a devastating and disabling condition that predominantly affecting younger population. Several management protocols have been suggested to improve outcome of acute traumatic SCI including conservative and operative treatment. For the operative treatment, surgical decompression in SCI could be achieved by posterior, posterolateral and anterior approaches. This systematic review aims to compare the approaches in performing decompression in spinal cord injury.

Methods: We conduct study from their inception dates to January 2021 with operative treatment of SCI with anterior and posterior approach as the inclusion criteria. Boolean method and PRISMA guideline was used to optimize the search and finding the study. All of the authors assessed the quality of study.

Results: A total of 4 studies were included in this systematic review. Three studies reported no significant difference between two approaches while one study described anterior approach is more effective than posterior approach.

Discussion: There is still much debate about the approach to be used in patients with spinal cord injuries. Three studies mentioned neither the anterior approach nor the posterior approach had significant differences in managing SCI operatively. One study reported neurological recovery was found to be better in patients operated with the anterior approach.
\end{abstract}

Conclusion: The effectiveness between the use of anterior and posterior approaches in patients with spinal cord injury that both had the same clinical outcome. The location of the difference is only in the posterior approach where there is a significant amount of blood loss also has a much longer hospital stay than the anterior approach

Keywords: Spinal Cord Injury, SCI, Surgical Approach, Anterior Approaches, Posterior Approaches

\section{INTRODUCTION}

Spinal cord injury (SCI) is a devastating and disabling condition that predominantly affecting younger population. It accounts for 16 per million population in Western Europe and 750 per million worldwide. The most common cause of SCI is trauma, but other factors such as tumor, infection, vascular lesions, or iatrogenic procedures can also cause this injury. Injury to the spinal column usually occurs at the cervicothoracic or thoracolumbar region. Injury to the spinal cord often irreversible due to primary and secondary process of injury, led to functional impact of the patient as well as economical burden to the society. (Cristante et al., 2012; Rath and Balain, 2017)

Pathophysiology of SCI consists of primary and secondary mechanism that damages the spinal cord. The primary process because of rapid direct compression and contusion of the cord, which initiates to inflammatory response. The secondary process results from biochemical and cellular processes that triggered by the primary lesion, gradually result in neuronal 
death. (Dalbayrak et al., 2015) Clinical manifestation of SCI varies depending on the site of injury, and the type of tract of the spinal cord. Classification of injury that commonly used is American Spinal Injury Association impairment scale (ASIA), a modification of Frankel classification that divide the injury into five classes depends on the severity of injury. (Rath and Balain, 2017)

Several management protocols have been suggested to improve outcome of acute traumatic SCI. consist of conservative and operative treatment. Methylprednisolone therapy that based on National Acute Spinal Cord Injury Studies (NASCIS), is one of the most used conservative treatment protocols. Methylprednisolone has potent antiinflammatory effect. This effect is predicted to reduce the secondary injury in SCI, which happens in effect to pro-inflammatory cytokine being released after trauma to the spinal cord. (Fehlings et al., 2017) The others conservative treatment includes biological therapy and physical therapy. (Cristante et al., 2012) Operative treatment of acute SCI decided based on spinal compression and neurological deficit that occur. Absolute indications for surgery in acute SCI patients include progressive neurological deficit in the presence of cord compression and dislocation type injury to the spinal column. Surgery is usually performed in the first 24 hours, or after 4-6 weeks in order to prevent secondary cord damage. (Rath and Balain, 2017)

Surgical decompression in SCI could be achieved by posterior, posterolateral and anterior approaches. Generally, the main approach for the patients without the presence of any pathology causing compression in the canal is the posterior stabilization and fusion. Anterior decompression and fusion as well as posterior stabilization are required for the patients with certain pathologies causing compression in the canal. Although, anterior or posterior surgical approach does not have any difference in some cases, in terms of fusion rates, sagittal alignment and neurological recovery. (Dalbayrak et al., 2015)

\section{MATERIAL AND METHODS Search Strategy}

Focused literature searches were primarily conducted using the Google Scholar and Pubmed/MEDLINE, from their inception dates to January 2021. To optimize sensitivity and specificity of the search method and identify all research, using the keywords listed below in combination with Boolean "AND" and "OR" phrases. Search terms: "spinal cord injury", "management" "surgical approach", "anterior approach", "posterior approach". A reference list of all included studies was identified as potentially relevant. The studies were included only in humans, and were written in English.

\section{Inclusion Criteria}

One reviewer screened the search results. Operative management of patients with spinal cord injury with anterior approach, posterior approach in this systematic review. Patients without spinal cord injury and nonoperative management were excluded. Other than studies using English were excluded in this systematic review.

\section{Quality Evaluation}

This was done, based on inclusion criteria the authors filtered eligible studies through titles and abstracts. Then, the authors screened the complete studies from all the studies that were collected. The author is looking for publications that are very relevant to be included in this research. The author also assessed the quality of the study. 
I Dewa Gede Bracika Damma Prasada et.al. Comparison of the anterior and posterior approaches for the management of spinal cord injury: a systematic review

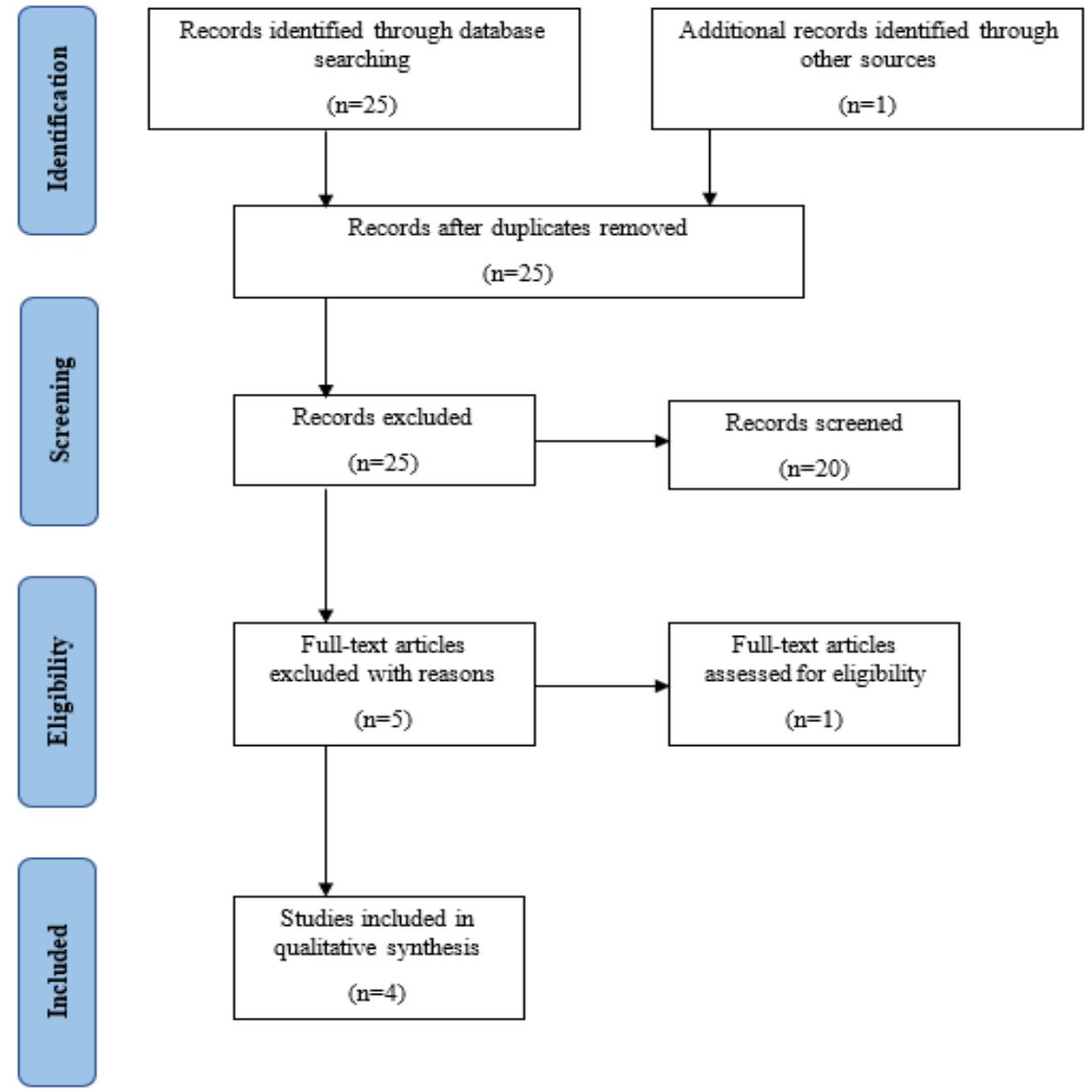

Figure1. Flow diagram based on PRISMA guideline.

\section{RESULTS}

The electronic search returned 52 records, after removing duplicate results. On the basis of screening of abstracts and titles, a total of 50 records were excluded. The remaining articles were studied by the authors independently based on the extracted full text. This selection process resulted in the final four articles for inclusion in the systematic review and is depicted in Figure 1.

In a study reported by Ren et. al. management of patients with spinal cord injury using either the anterior approach or the posterior approach did not show any significant difference in terms of recovery period $(65.5 \pm 89.6 \%$ vs $64.7 \pm 54.5 \%$; $\mathrm{P}=$ 0.951). The difference in ASIA (American Spinal Injury Association) scores using the anterior approach or using the posterior approach was no significant difference either before surgery, at 6 months or two years postoperatively. The anterior approach and the posterior approach did not have a significant difference ( $\mathrm{P}>$ 0.05). Study conducted by Ren et. al also reported that the posterior approach had more blood loss than the anterior approach $(\mathrm{P}<0.001)$ and that the posterior approach also had a significantly longer hospital stay $(\mathrm{P}<0.001)$ (Ren et al., 2020).

In the study reported by Dalbayrak et al, it was found that the management of patients with spinal cord injury using the anterior approach, namely the anterior decompression is more effective than the posterior approach, namely posterior decompression. What was also found was that neurological recovery was found to be better in patients operated on with the anterior approach. In addition, Dalbayrak et al also reported that anterior decompression was easier to apply for patients with burst fractures, whereas no difference was found between the groups in terms of sagittal alignment (Dalbayrak et al., 2015) 
In the study reported by Liu et al, management of patients with spinal cord injury using the anterior approach, namely corpectomies or using the posterior approach, namely laminectomy, did not report any complications. In addition, Liu et al also reported that the management of patients with spinal cord using the anterior and posterior approaches both had ideal results in terms of functional outcomes and radiological comparisons (Liu et al., 2012).

In the study reported by Brodke et al, management of patients with spinal cord injury using the anterior approach and the posterior approach did not have a significant difference in terms of neurologic outcome $(\mathrm{P}=0.54)$. Ten of the 20 patients $(50 \%)$ in the anterior group had complete spinal cord injuries (Frankel A) preoperatively, dropping to $6(30 \%)$ postoperatively. In the posterior group, 16 of the 27 patients $(59 \%)$ were complete preoperatively, dropping to $10(37 \%)$ postoperatively. Again, there was no statistically significant difference (Brodke et al., 2003). Apart from that from the study conducted by Brodke et al, there was no significant difference in the number of complications between the anterior and posterior groups. In the anterior group, one patient had pneumonia, one patient had adult respiratory distress syndrome, and there was one instrumentation failure. There were also two patient deaths due to unrelated causes. In the posterior group, two patients developed pneumonia and one instrumentation failure, although these patients continued to experience fusion. There were seven patients in each group who reported neck pain at the last follow-up (Brodke et al., 2003).

\section{DISCUSSION}

In this discussion, we will discuss the use of the anterior approach and the posterior approach in spinal cord management. There is still much debate about the approach to be used in patients with spinal cord injuries. Although many improvements have been made in the surgical treatment of spinal cord injuries, another issue is debated regarding the optimal timing for surgical intervention. Several studies related to the surgical approach performed in patients with spinal cord injuries.

In a study conducted by Ren et.al the anterior approach and the posterior approach in patients with spinal cord injury did not have a significant difference in terms of both recovery period and clinical outcome. However, on the other hand, the posterior approach has a lot of blood loss during surgery and also has a much longer hospital stay compared to the anterior approach. (Ren et al., 2020)

In a study reported by Dalbayrak et al. Anterior decompression is more effective than the posterior approach, namely posterior decompression. What was also found was that neurological recovery was found to be better in patients operated on with the anterior approach. In addition, anterior decompression was easier to apply for patients with burst fractures whereas no difference was found between the groups in terms of sagittal alignment (Dalbayrak et al., 2015).

In the study reported by Liu et al, the use of corpectomies as an anterior approach in patients with spinal cord injury and laminectomy as a posterior approach did not report any complications and both had ideal results in terms of functional outcome and radiological comparisons. (Liu et al., 2012)

In the study reported by Brodke et al, neither the anterior approach nor the posterior approach had significant differences in terms of neurologic outcome and complications between the anterior and posterior groups. In the anterior group, one patient had pneumonia, one patient had adult respiratory distress syndrome, and there was one instrumentation failure. There were also two patient deaths due to unrelated causes. In the posterior group, two patients developed pneumonia and one instrumentation failure, although these patients continued to experience fusion. There were seven patients in each group 
who reported neck pain at the last follow-up (Brodke et al., 2003).

There is still much debate about the approach to be used in patients with spinal cord injuries. Although many improvements have been made in the surgical treatment of spinal cord injuries, another issue is debated regarding the optimal timing for surgical intervention. Further research on the approach used and the operating time is needed.

\section{CONCLUSIONS}

The management of patients with spinal cord injury is still quite debated, both with operative and non-operative management. In addition, the exact timing of surgery in patients with spinal cord injuries is still being debated. From this systematic review, it was found that the effectiveness between the use of anterior and posterior approaches in patients with spinal cord injury that both had the same clinical outcome. The location of the difference is only in the posterior approach where there is a significant amount of blood loss also has a much longer hospital stay than the anterior approach.

\section{Acknowledgement: None}

Disclosure of interest: The authors declare that they have no conflicts of interest concerning this article.

Source of Funding: The authors received no financial support for the research, authorship, and/or publication of this article.

\section{REFERENCES}

1. Brodke DS, Anderson PA, Newell DW, et al. (2003) Comparison of anterior and posterior approaches in cervical spinal cord injuries. Journal of Spinal Disorders and Techniques. DOI: 10.1097/00024720200306000-00001.

2. Cristante AF, de Barros Filho TEP, Marcon RM, et al. (2012) Therapeutic approaches for spinal cord injury. Clinics. DOI: 10.6061/clinics/2012(10)16.
3. Dalbayrak S, Yaman $\mathrm{O}$ and Yilmaz $\mathrm{T}$ (2015) Current and future surgery strategies for spinal cord injuries. World Journal of Orthopaedics. DOI: 10.5312/wjo.v6.i1.34.

4. Fehlings MG, Wilson JR, Harrop JS, et al. (2017) Efficacy and Safety of Methylprednisolone Sodium Succinate in Acute Spinal Cord Injury: A Systematic Review. Global Spine Journal. DOI: 10.1177/2192568217706366.

5. Liu B, Ma W, Zhu F, et al. (2012) Comparison between anterior and posterior decompression for cervical spondylotic myelopathy: subjective evaluation and cost analysis. Orthopaedic surgery. DOI: 10.1111/j.1757-7861.2011.00169.x.

6. Rath N and Balain B (2017) Spinal cord injury-The role of surgical treatment for neurological improvement. Journal of Clinical Orthopaedics and Trauma. DOI: 10.1016/j.jcot.2017.06.016.

7. Ren C, Qin R, Wang Peng, et al. (2020) Comparison of anterior and posterior approaches for treatment of traumatic cervical dislocation combined with spinal cord injury: Minimum 10-year follow-up. Scientific Reports. DOI: 10.1038/s41598020-67265-2.

8. Brodke DS, Anderson PA, Newell DW, et al. (2003) Comparison of anterior and posterior approaches in cervical spinal cord injuries. Journal of Spinal Disorders and Techniques. DOI: 10.1097/00024720200306000-00001.

9. Cristante AF, de Barros Filho TEP, Marcon RM, et al. (2012) Therapeutic approaches for spinal cord injury. Clinics. DOI: 10.6061/clinics/2012(10)16.

10. Dalbayrak S, Yaman $O$ and Yilmaz $T$ (2015) Current and future surgery strategies for spinal cord injuries. World Journal of Orthopaedics. DOI: 10.5312/wjo.v6.i1.34.

11. Fehlings MG, Wilson JR, Harrop JS, et al. (2017) Efficacy and Safety of Methylprednisolone Sodium Succinate in Acute Spinal Cord Injury: A Systematic Review. Global Spine Journal. DOI: 10.1177/2192568217706366.

12. Liu B, Ma W, Zhu F, et al. (2012) Comparison between anterior and posterior decompression for cervical spondylotic myelopathy: subjective evaluation and cost analysis. Orthopaedic surgery. DOI: 10.1111/j.1757-7861.2011.00169.x. 
I Dewa Gede Bracika Damma Prasada et.al. Comparison of the anterior and posterior approaches for the management of spinal cord injury: a systematic review

13. Rath N and Balain B (2017) Spinal cord injury-The role of surgical treatment for neurological improvement. Journal of Clinical Orthopaedics and Trauma. DOI: 10.1016/j.jcot.2017.06.016.

14. Ren C, Qin R, Wang Peng, et al. (2020) Comparison of anterior and posterior approaches for treatment of traumatic cervical dislocation combined with spinal cord injury: Minimum 10-year follow-up.
Scientific Reports. DOI: 10.1038/s41598020-67265-2.

How to cite this article: I Dewa Gede Bracika Damma Prasada, Ketut Gede Mulyadi Ridia. Comparison of the anterior and posterior approaches for the management of spinal cord injury: a systematic review. International Journal of Science \& Healthcare Research. 2021; 6(3): 333-338. DOI: https://doi.org/ 10.52403/ijshr.20210757 